\title{
Test mode of operation network of monitoring subsoil radon in the south of Sakhalin
}

\author{
Evgeny Makarov ${ }^{1,2,}{ }^{*}$, Pavel Firstov ${ }^{1}$, Dmitry Kostylev ${ }^{3,5}$, Evgeny Rylov ${ }^{4}$, and Ilya \\ Dudchenko ${ }^{5}$ \\ ${ }^{1}$ Kamchatka Branch, Geophysical Survey, Russian Academy of Sciences, Petropavlovsk- \\ Kamchatsky, Russia \\ ${ }^{2}$ Kamchatka State University named after Vitus Bering, Petropavlovsk-Kamchatsky, Russia \\ ${ }^{3}$ Sakhalin Branch, Geophysical Survey, Russian Academy of Sciences, Yuzhno-Sakhalinsk, Russia \\ ${ }^{4}$ Institute of Volcanology and Seismology, Far East Branch, Russian Academy of Sciences, \\ Petropavlovsk-Kamchatsky, Russia \\ ${ }^{5}$ Institute of Marine Geology and Geophysics, Far East Branch, Russian Academy of Sciences, \\ Yuzhno-Sakhalinsk, Russia
}

\begin{abstract}
Radon monitoring in the literature is recognized as a promising method for the prediction of earthquakes. In the last decade, the promise of the radon method for predicting subduction earthquakes has been convincingly demonstrated on Kamchatka. The results obtained on the Kamchatka, give reason to hope for the detection of predictive anomalies in the radon field and for weaker, but not less dangerous, small-focus earthquakes on the Sakhalin Island. The southern part of Sakhalin Island is a region of high seismic hazard. On Sakhalin Island, in a test mode, a network of three radon monitoring stations in the subsoil air is launched using the forced convection method to search for earthquake precursors. Based on the literature data, it is assumed that the effectiveness of radon monitoring near the focal zone of small-focus earthquakes on Sakhalin Island will be significantly higher compared to the conditions of the subduction zone in Kamchatka. Data from the network of radon monitoring stations can be used as an additional parameter to substantiate the conclusions on possible scenarios for the evolution of the seismic process in the south of Sakhalin.
\end{abstract}

\section{Introduction}

The prospectivity of the seismoemanation method for the purpose of earthquake prediction is shown in numerous publications, references to which can be found in survey papers [Zubkov, 1981; Dubinchuk, 1991; Cicerone at al., 2009].

In the last decade, the promise of the radon method for predicting subduction earthquakes has been convincingly demonstrated in Kamchatka [Firstov, Makarov, 2015, Firstov et al., 2018]. Since 1997, at the Petropavlovsk-Kamchatsky geodynamic range, work is underway to measure the volume activity of subsoil radon (SR). For $67 \%$ of earthquakes with $5.5<\mathrm{M}<6.9$ with epicenters in the subduction zone at a distance of more

\footnotetext{
* Corresponding author: ice@emsd.ru
} 
than $100 \mathrm{~km}$ from the registration points, predictive anomalies in the SR are identified with a lead time from several days to several hours. The optimistic results obtained in Kamchatka give us hope for the discovery of predictive anomalies in the field of SR and for weaker, but not less dangerous, small-focus earthquakes of Sakhalin Island.

The southern part of Sakhalin Island is a region of high seismic hazard (Fig. 1). There are 4 earthquakes, the intensity of shaking from which in nearby settlements exceeded 7 points: Moneron, September 5, 1971, $M=7.2$; Takoy, September 1, 2001, with $\mathrm{M}=5.5$; Gornozavodsk, August 17, 2006 with $\mathrm{M}=5.6$; Nevelsk, August 2, 2007 with $\mathrm{M}=6.1$.

The active development of hydrocarbon production and transportation systems to the ports of shipment on Sakhalin Island increases the risk of seismic hazard. Therefore, seismic monitoring and development of methods that contribute to increasing the effectiveness of earthquake prediction is an urgent task for Sakhalin Island. The urgency of this work is conditioned, in addition to the above, by the fact that according to the conclusions of the Sakhalin branch of the Russian Expert Council on the forecast of earthquakes, the assessment of seismic hazard and risk (SF RES), a medium-term earthquake forecast with a magnitude $\mathrm{M}=6.0+/-0.7$.

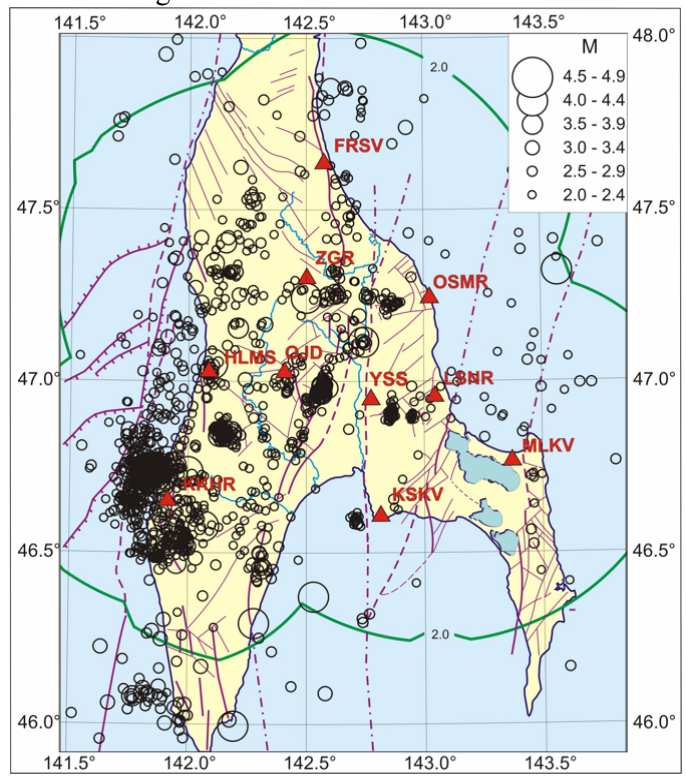

Fig. 1. Map of epicenters of earthquakes in the area of southern Sakhalin with a magnitude $M \geq 2.0$ for 2012-2014. The zone of reliable registration of earthquakes is shown by a green line. The purple line shows the faults, red triangles marked seismic stations: YSS - Yuzhno-Sakhalinsk; ZGR Zagorskoye; KKHR - Kolkhoznoye; KSKV - Korsakov; LSNR - Lesnoye; MLKV - Malkovo; OJD Ozhidayevo; STRD - Starodubskoye; FRSV - Firsovo; HLMS - Kholmsk.

Kamchatka Branch of the Russian Academy of Sciences (KB GS RAS) jointly with the Institute of Marine Geology and Geophysics of the Far Eastern Branch of the RAS (IMGG FEB RAS) and the Sakhalin Branch of the Russian Academy of Sciences (SB GS RAS) on Sakhalin Island in the summer of 2018 work was done on the organization and launch of a network of three monitoring stations in a test mode. The coordinates of the points are given in Tab. 1, and their arrangement is shown in Fig. 2. 


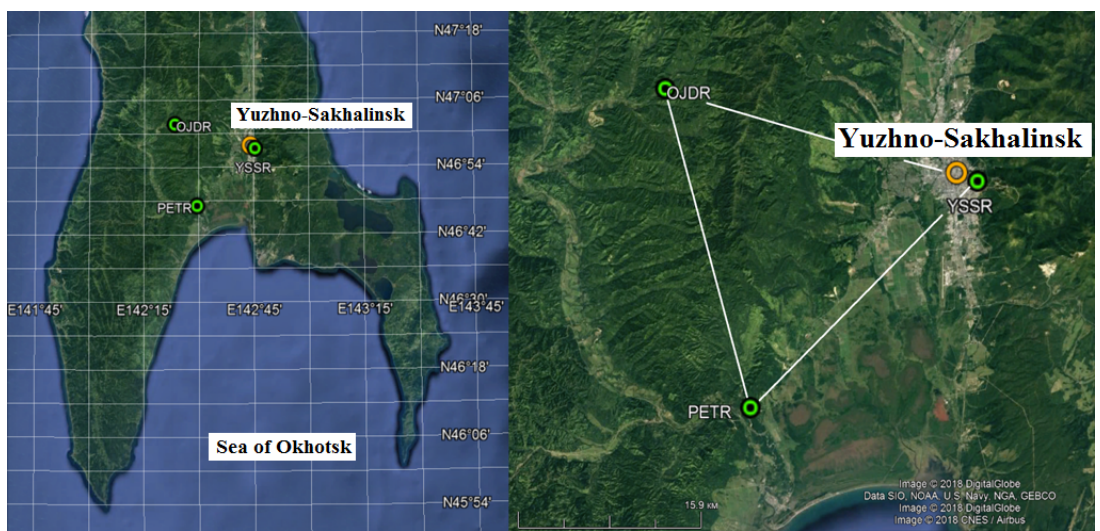

Fig. 2. Scheme of location of monitoring stations for subsoil radon concentration in the south of Sakhalin Island. YSSR is a point in Yuzhno-Sakhalinsk (territory of the SB GS RAS); PETR - point in Petropavlovskoye; OJDR point in Ozhidayevo.

The points were located in the corners of an equilateral triangle with a side of $\sim 30 \mathrm{~km}$. In the works [Firstov, Makarov, 2015; Firstov et al., 2018; Makarov, 2017] showed that the area arrangement of instruments makes it possible to detect disturbances of SR with a duration of several days and with a lead time of 0.5-12 days before earthquakes with $\mathrm{M}>$ 5.5 of the Avachinsky Bay area, which are associated with the passage through the terrestrial medium of signals called by the authors «deformation waves» (DW), which can arise as a result of quasi-plastic flow of geomaterial at the last stage of the preparation of strong earthquakes. The apparent speed of their distribution according to the network of monitoring stations SR in Kamchatka is estimated at $14-144 \mathrm{~km} /$ day. According to the authors, the anomalies in the dynamics of the SR are formed as a result of the passage of the soliton type DW, which, acting on the emanating horizon at the registration points, change the value of $\mathrm{AC} \mathrm{Rn}$ in the aeration zone.

Table 1. Parameters of points of the network of continuous monitoring of SR in the south of. Sakhalin.

\begin{tabular}{|c|c|c|c|c|c|}
\hline \multirow{2}{*}{$\begin{array}{c}\text { Date of } \\
\text { beginning of } \\
\text { observations }\end{array}$} & \multicolumn{2}{|c|}{ Coordinates } & \multirow{2}{*}{$\begin{array}{l}\text { Item } \\
\text { name }\end{array}$} & \multirow{2}{*}{$\begin{array}{l}\text { SR in the } \\
\text { ground, } \\
\mathrm{Bq} / \mathrm{m}^{3}\end{array}$} & \multirow{2}{*}{ Note } \\
\hline & $\mathbf{N}$, lat & E, lon & & & \\
\hline $02 / 07 / 2018$ & $46^{\circ} 47^{\prime} 19.00^{\prime \prime}$ & $142^{\circ} 29^{\prime} 45.58^{\prime \prime}$ & PETR & 210 & Depth of the hole $1 \mathrm{~m}$ \\
\hline $05 / 07 / 2018$ & $47^{\circ} 01^{\prime} 50.25^{\prime \prime}$ & $142^{\circ} 23^{\prime} 41.61^{\prime \prime}$ & OJDR & 17300 & Depth of the hole $1,7 \mathrm{~m}$ \\
\hline $06 / 07 / 2018$ & $46^{\circ} 57^{\prime} 31.01^{\prime \prime}$ & $142^{\circ} 45^{\prime} 37.04^{\prime \prime}$ & YSSR & 7300 & Depth of the hole $2 \mathrm{~m}$ \\
\hline
\end{tabular}

\section{Methodology of observations}

As devices for continuous monitoring of the SR, radiometers RADEX MR107 manufactured by the Russian company KVARTA-RAD LLC, which is a modern semiconductor device assembled in a single package and equipped with control elements and a screen, were chosen. This radiometer is designed to evaluate the equivalent of the equilibrium volume activity of $\mathrm{Rn}$ and the daughter products of radon isotopes in terms of volume Rn activity in the air of residential and public premises. RADEX MR107 allows you to analyze the dynamics of changes, signal the excess of the permitted sanitary standards for radon in the air of the premises, and also transfer the measured results to a personal computer. In addition to measuring $\mathrm{Rn}$ by the diffusion method, the device 
registers both air temperature and humidity. All received data is stored in a long-term independent memory and transferred to the $\mathrm{PC}$ at the request of the operator with the help of special software from the package. Control of measurement modes, adjustment of the date and time is carried out with the help of a PC.

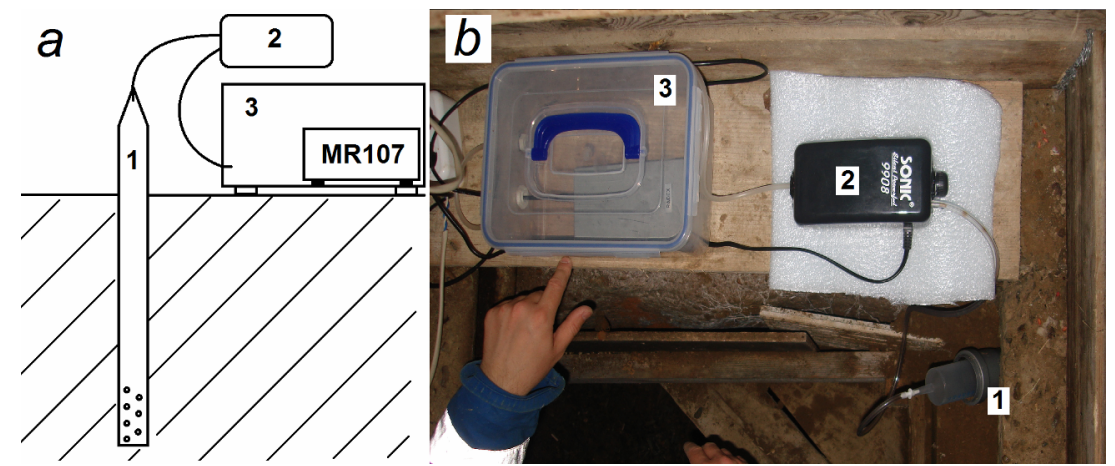

Fig. 3. Block diagram (a) and general view (b) of the subsoil radon registration kit. 1 - perforated pipe in the hole; 2 - the compressor; 3 - storage chamber with the device MR107.

For the organization of observations using the MR107 devices, the forced convection method was used [Utkin, Yurkov, 2010]. The essence of the method consists in evacuating subsoil air containing $\mathrm{Rn}$ from the measuring hole with the help of a compressor to the storage chamber where the device is installed (Fig. 3). As is known, the main interference in the measurement of volumetric activity of SR are the variations in meteorological values: air temperature, atmospheric pressure, soil moisture [Rudakov, 2009; Kozlova, Yurkov, 2005]. The suggested measurement technique with pumping out the subterranean air from the measuring hole can significantly reduce the level of these interference [Utkin, Yurkov, 2010].

\section{Description of monitoring points for SR}

Points of monitoring SR were installed in the South of Sakhalin in the Central Sakhalin fault zone, which includes faults along the western boundaries of the main intermontane depressions of Sakhalin - Susunay and Tym-Poronaysk. This zone, sometimes called the Tym-Poronaysk active zone, includes the Klyuchevskoy fault (Tym-Poronaysk depression) and the Aprelovsky fault in the Susunay depression. [Kozhurin, Kim, 2010].

The kinematics of the Central Sakhalin fault zone as a whole is characterized by an overthrust. The seismic activity of the fault zone is currently not high. However, based on the results of paleoseismological studies, the fault can generate earthquakes with $\mathrm{M}=7.0$ 7.5 [Streltsov, Kozhurin, 2002]. In Fig. 4 shows the faults and tectonic disturbances of the southern part of Sakhalin from [Rozhdestvensky, Saprygin, 1999], as well as the location of the points with respect to tectonic faults.

In the locations of SR monitoring stations, seismic surveys and emanation surveys were conducted to study the structure of the upper part of the geological section and to determine the points of installation of radiometers.

The PETR point was located in the area of the temporary seismic station PET IMGG FEB RAS at an elevation in the basement of a residential building in the valley of the Liutoga River (Petropavlovskoye settlement). Subflow of subsoil air was carried out from a depth of one meter. At the time of registration, the activity concentration of radon (AC Rn), measured with a RGA-01 radiometer, was $0.21 \mathrm{kBq} / \mathrm{m}^{3}$. 
The OJDR point in Ozhidayevo village was located in the seismic station OJD SB GS RAS in the floodplain of the river Ozhidayevskaya. Submergence of subterranean air was carried out in the basement of a residential building from a depth of $1.7 \mathrm{~m}$. At the time of the start of registration, the AC Rn was $17.3 \mathrm{kBq} / \mathrm{m}^{3}$. Such high values of AC Rn in the air were not observed in the subsoil even during the emanation survey of the Volchanskoye thermal water deposit area [Chelnokov et al., 2015]. It is not excluded that this point is confined to the fault zone and this gives hope for its high strain-sensitivity.

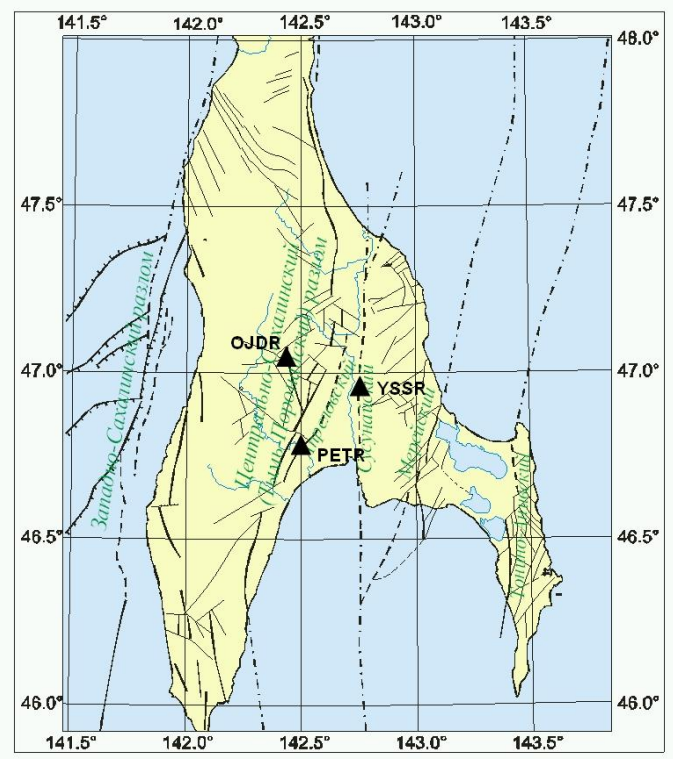

Fig. 4. The position of the points of continuous monitoring of the SR and the breaking tectonics of the south of Sakhalin according to V.S. Rozhdestvensky and S.M. Saprygin [1999].

The YSSR point was located on the territory of SB GS RAS on the slope of the hill near the installation of the seismic station YSS. Subsoil air was pumped from a depth of two meters. At the time of registration, AC Rn was $7.3 \mathrm{kBq} / \mathrm{m}^{3}$.

\section{The results of the network operation of the registration points of the SR in the test mode}

The results of the work from July 2 to August 20 are shown in Fig. 5. During this period the equipment worked stably and without failures. It should be noted, reliable operation of the entire complex, including when the mains power is disconnected. The moment the compressor turns off when the power supply is cut off at the OJDR point is shown in Fig. 5 with a gray arrow.

Further analysis of the obtained data and comparison with the seismicity of the region will make it possible to detect abnormal changes in the AC Rn that precede earthquakes. For the identified anomalies, similar to the Kamchatka anomalies, based on the accumulated experience and experimental data on morphological features, the main types will be singled out [Makarov, 2017; Firstov et al., 2018] and compared them with data from literature sources. 

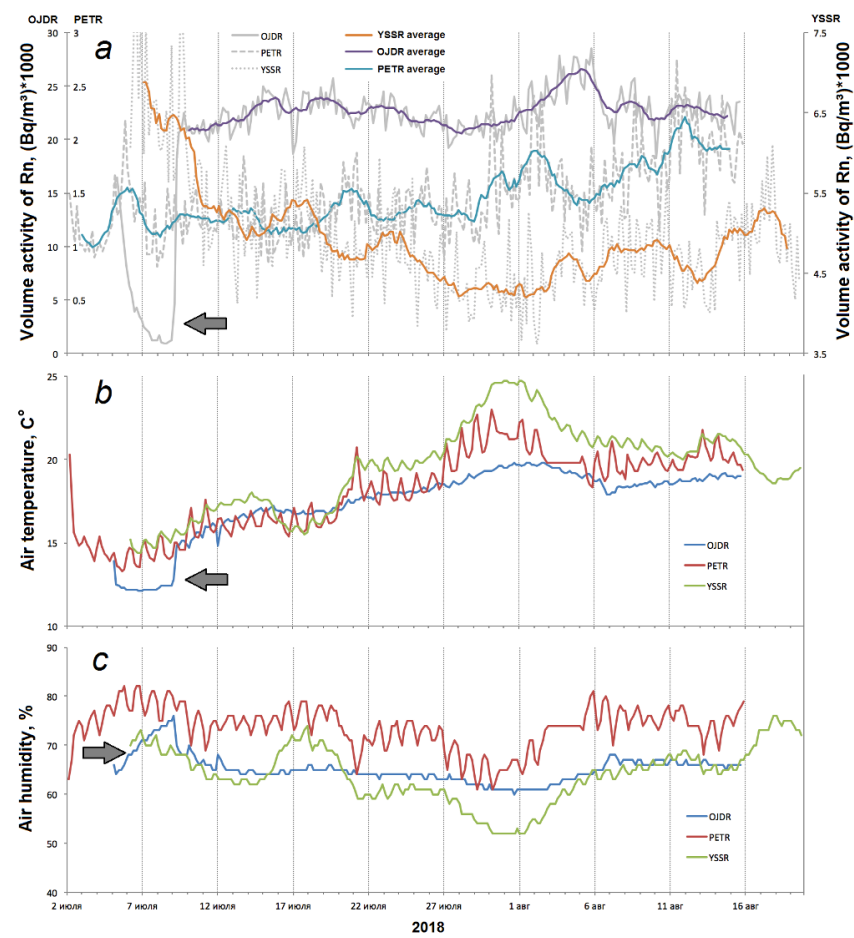

Fig. 5. Dynamics of AC Rn (a), temperature (b) and humidity (c) of subsoil air at the points of Sakhalin Island for the period July 2 - August 20, 2018, working in the test mode.

The experience of the Kamchatka subsoil gas monitoring network shows that the amplitude of anomalous perturbations preceding earthquakes in the subsoil $\mathrm{Rn}$ field is in the range $\delta=18-500 \%$, which is often less than the interference amplitudes reaching $300 \%$ and associated with the impact of meteorological values. Therefore, it is extremely important for expert evaluation and allocation of anomalies in SR dynamics preceding earthquakes, against the background of regularly occurring changes in the dynamics of subsoil gases associated with other processes, is the detection of in-phase variations at several registration points. Equally important is the search for optimal methods to reduce the impact of meteorological factors and ensure reliable operation of the network without losing data during long-term continuous registration.

\section{Conclusion}

The installation of radiometers on Sakhalin Island can be located in close proximity to seismically active areas (Fig. 1), which can not be realized for the subduction zone in the region of the Kamchatka Peninsula. Investigations of the SR as an indicator of the stressstrain state of the geo-environment on the network of stations on Sakhalin Island are promising and necessary for understanding the mechanism of the formation of predictive anomalies in the field of SR for crustal earthquakes. In the case of creating a network of monitoring points for AC $\mathrm{Rn}$ in Southern Sakhalin and obtaining data with predictive anomalies, their comparison with the data of the Petropavlovsk-Kamchatsky landfill will 
help to study more deeply the processes associated with the change in the stresseddeformed state of the geo-environment at the last stage of the preparation of strong earthquakes.

Based on the literature data, it is assumed that the effectiveness of radon monitoring near the focal zone (shalow-focus earthquakes), which is typical for the southern part of Sakhalin Island, will be significantly higher than the conditions of the subduction zone in Kamchatka. Data from the network of monitoring stations of the SR can be used as an additional parameter to substantiate the conclusions on possible scenarios for the development of the seismic process in the south of Sakhalin.

The work was carried out with the financial support of the RFBR grant No.16-0500162.

\section{References}

1. S.I. Zubkov, Journal of Volcanology and Seismology 6, 74-105 (1981)

2. I.A. Kozlova, A.K.Yurkov, Ural Geophysical Gazette 7, 31-34 (2005)

3. A.I. Kozhurin, Kim Chun Un, Active faults on. Sakhalin, estimation of magnitude and repeatability of the maximum possible earthquakes (ITG FEB RAS, 2010)

4. E.O. Makarov, Abstract (Petropavlovsk-Kamchatsky, 2017)

5. V.P. Rudakov, Emanational monitoring of geomethods and processes (Scientific World, 2009)

6. M.I. Streltsov, A.I. Kozhurin, Active faults and catastrophic earthquakes in Sakhalin (IMGG FEB RAS, 2002)

7. V.S. Rozhdestvensky, S.M. Saprygin, Russian Journal of Pacific Geology 6, 59-70 (1999)

8. V.I. Utkin, A.K.Yurkov, Russian Geology and Geophysics 2, 277-286 (2010)

9. P.P. Firstov, E.O. Makarov, R.R. Akhashev, Seismic Instruments 1, 60-80 (2015)

10. P.P. Firstov, E.O. Makarov, Seismic Instruments 4, 58-80 (2015)

11. P.P. Firstov, E.O. Makarov, I.P. Glukhova, D.I. Budilov, D.V. Isakevich, Geosystems of Transition Zones 1, 16-32 (2108)

12. R.D. Cicerone, J.E. Ebel, J.A. Beitton, Tectonophysics 476, 371-196 (2009)

13. G. Chelnokov, R. Zharkov, I. Bragin, Journal of geoscience and environment protection 3, 48-53 (2015)

14. V.T. Dubinchuk, Radon as a precursor of earthquakes (Vienna, 1991) 